Original Research Paper

\title{
Enhanced Preemptive Global Utility Accrual Real Time Scheduling Algorithms in Multicore Environment
}

\author{
Idawaty Ahmad, Mohamed Othman and Zuriati Ahmad Zulkarnain \\ Department of Communication Technology and Network, Faculty of Computer Science and Information Technology, \\ University Putra Malaysia, 43400 UPM, Serdang, Selangor DE, Malaysia
}

\author{
Article history \\ Received: 05-11-2015 \\ Revised: 09-02-2016 \\ Accepted: 10-02-2016 \\ Corresponding Author: \\ Idawaty Ahmad \\ Department of Communication \\ Technology and Network, \\ Faculty of Computer Science \\ and Information Technology, \\ University Putra Malaysia, \\ 43400 UPM, Serdang, Selangor \\ DE, Malaysia \\ Email: idawaty@upm.edu.my
}

\begin{abstract}
This paper proposed an efficient real time scheduling algorithm using global scheduling paradigm running in multicore environment known as Global Preemptive Utility Accrual Scheduling (GPUAS) algorithm. The existing TUF/UA multiprocessor scheduling algorithms known as GreedyGlobal Utility Accrual (G-GUA) and Non Greedy-Global Utility Accrual (NG-GUA) algorithms is seen to overlook the efficiency on its task scheduling algorithm. These algorithms have adapted the task migration attribute considering the load balancing problem in multi core platform. The existing PUAS uniprocessor scheduling algorithm is mapped into the multicore scheduling environment that consists of the global scheduling schemes considering the migration attribute of the executed tasks. The main principal of global scheduling is that it allows the executed tasks to migrate from one processor to the other processors whenever a scheduling event occurs in the system. The proposed GPUAS algorithm inherits the characteristics of PUAS in uniprocessor where it can preempt the highest PUD task at any event that occurs in the system. In this research, the proposed GPUAS algorithm enhanced the existing NG-GUA and G-GUA algorithms. The developed simulator has derived the set of parameter, events and performance metrics according to a detailed analysis of the base model. The proposed GPUAS algorithm achieved the highest accrued utility for the entire load range. The proposed GPUAS algorithm is more efficient than the existing algorithms, producing the highest accrued utility ratio and less abortion ratio making it more suitable and efficient for real time application domain.
\end{abstract}

Keywords: Real Time System, Utility Accrual Scheduling, Multicore, Discrete Event Simulation

\section{Introduction}

In the presence of extremely overloaded tasks traffic, the RTS requires multicore environment with an efficient load sharing capability to accommodate the surplus load. The load sharing mechanism is required in order to migrate the executed tasks across multiple processors. This ensures that no processor is idle while some tasks are waiting to be scheduled on other processors. The load sharing problem in multiprocessor environment can be solved by deploying the task migration attribute in the executed tasks.

\section{Problem Statement}

The existing TUF/UA multiprocessor scheduling algorithms known as Greedy-Global Utility Accrual
(G-GUA) and Non Greedy-Global Utility Accrual (NG-GUA) algorithms (Garyali et al., 2010) is seen to overlook the efficiency on its task scheduling in multicore platform. G-GUA uses a greedy strategy where task whose execution yields the maximum PUD is selected to be scheduled at a particular instance. For load sharing purpose, the requesting task is placed at the queue that has the least remaining execution cost. This requesting task assignment behavior in G-GUA may affect the total utility accrued because it does not consider the value of utility when determining the suitable queue at that particular instance. On the other hand, NG-GUA uses dual metric to overcome overloaded tasks in RTS. During under load it uses deadline and during overloaded condition, task whose execution yields the maximum PUD is selected to be 
scheduled at a particular instance. However, NG-GUA immediately aborts the requesting task that has potential to produce zero utility to the system due to its infeasibility. The abortion of a task leading to a zero utility acquired by the requesting task to the system. This will have an effect on the total utility accrued to the system. If the underlying scheduling scheme can reduce the abortion of the requesting task efficiently, then the system will possibly attain a higher utility thus enhancing the overall system's performance.

\section{Objective}

The objective of this research is to enhance the efficiency of the existing TUF/UA scheduling algorithms in multiprocessor environment i.e., NGGUA and G-GUA to accommodate the overloaded tasks traffic so that the maximum total utility accrued to the system. This paper proposed the GPUAS algorithm that considered an efficient task request location with migration task attribute for solving the overloaded situation. If the underlying scheduling scheme places the task's request among the processor's queue efficiently and reduce the abortion problems, then the system will gain higher utility thus enhancing the overall system's performance.

\section{Materials and Methods}

\section{Approach}

A discrete event simulation is used as a methodology to verify the performances of GPUAS and the existing algorithms. In order to precisely remodel and further enhance the algorithms, DES written in $\mathrm{C}$ language in Visual $\mathrm{C}++$ environment is the best method to achieve this objective. Figure 1 shows the deployed simulation framework.

\section{Simulation Framework}

The simulation of multicore infrastructure consists of a source, task entities and an array of utlist queues and resources to represent the various numbers of processors in the system.

\section{Task Model}

Tasks have one of two types of migration characteristics. The migration type of a task is denoted by Migration $\in\{$ NON MIGRATE, MIGRATE $\}$. Tasks that are not allowed to migrate among processors possess the NON_MIGRATE attribute while those with a MIGRATE attribute can be migrated among the processors and are considered only in the global scheduling paradigm.

\section{TUF Model}

The general dominant task attribute is associated to using timing constraint which is denoted as deadline.
The timing constraint of a task is designed using the step and arbitrary TUF model in this study (Li et al., 2006). A TUF describes a task utility contribution to the system as a function of its completion time. The TUF shape of a task is denoted by Shape $\in$ \{STEP, ARBITRARY $\}$. The step TUF model used in the simulator is shown in Fig. 2. The maximum utility that could possibly be gained by a task is denoted as MaxAU. The random value of MaxAU abides normal distribution $(10,10)$ i.e., the mean value and variance is set 10 to conform to the benchmark. The InitialTime is the starting time for which the function is defined. The TerminationTime is the last time for which the function is defined. That is, MaxAU is defined in within the time interval of [InitialTime, TerminationTime]. The completion of a task at an instance i.e., sclock within this interval will yield a random positive utility denoted as Utility which is equal to the MaxAU for step TUF model as shown in Fig. 2a. The completion of a task breaching the stipulated deadline causes the value of Utility and MaxAU to become zero. If the TerminationTime is reached and the task has not finished its execution, it accrues zero utility to the system.

The arbitrary shape TUF is represented as a continuous and derivable polynomial equation as shown in Fig. 2b. The maximum utility that could possibly be gained by a task is denoted as MaxAU. The random value of $M a x A U$ abides normal distribution $(10,10)$ i.e., the mean value and variance is set 10 to conform to the benchmark. For arbitrary TUF, the completion of a task within the InitialTime and TerminationTime interval will yield a random positive utility denoted as Utility as shown in Fig. 2 b.

\section{Task Assignment Algorithm}

All tasks are assigned to processors by task assignment algorithm as shown in Fig. 3. The number of processors is checked before the task is assigned to a specific processor. The Tgen.cpuid parameter is used to identify the assigned processor ID. In uniprocessor environment the value of Tgen.cpuid is equal to 0 . This indicates that the processor ID zero is assigned to task Tgen. In multiprocessor environment, the generated task Tgen is assigned according to the value of ShortestCPU parameter. This parameter captures the processor ID that has the smallest value of TotalExec[cpuid]] value in their respective cpuid queue. The TotalExec[cpuid] measures the execution time of all requests that are currently pending in the cpuid queue. The TotalExec parameter is increased every time a request of a task is inserted into the cpuid queue. The TotalExec parameter is reduced every time a request of a task is deleted from the cpuid queue. 


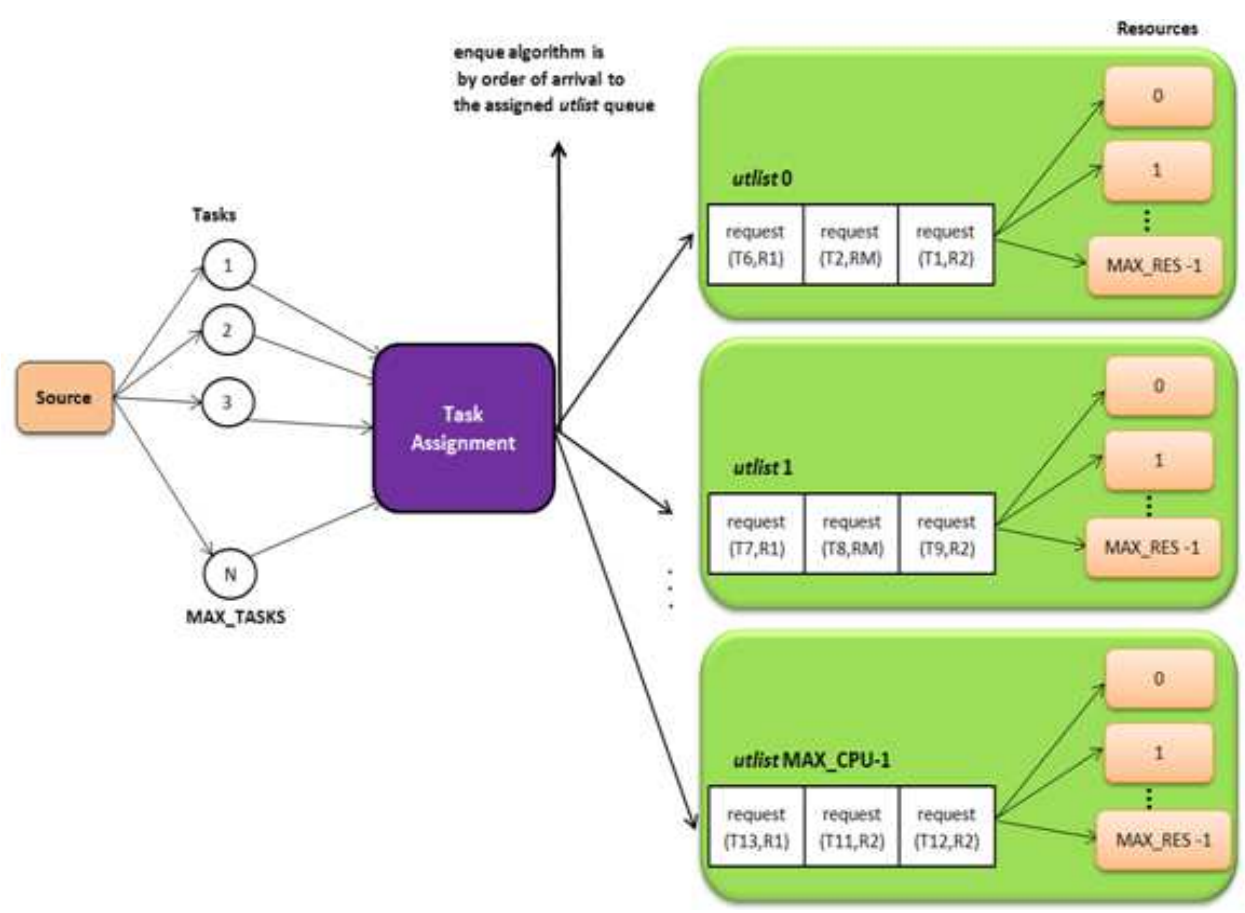

Fig. 1. Simulation framework (Idawaty et al., 2012)
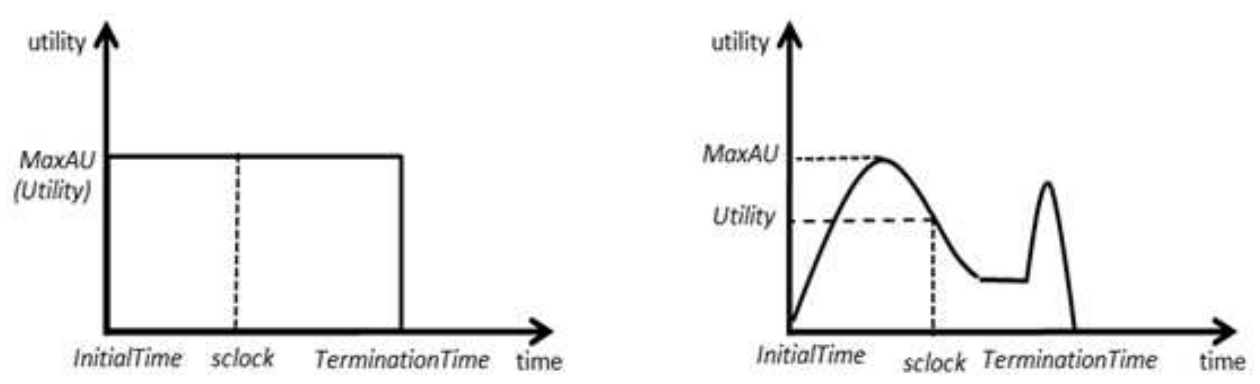

Fig. 2. TUF model

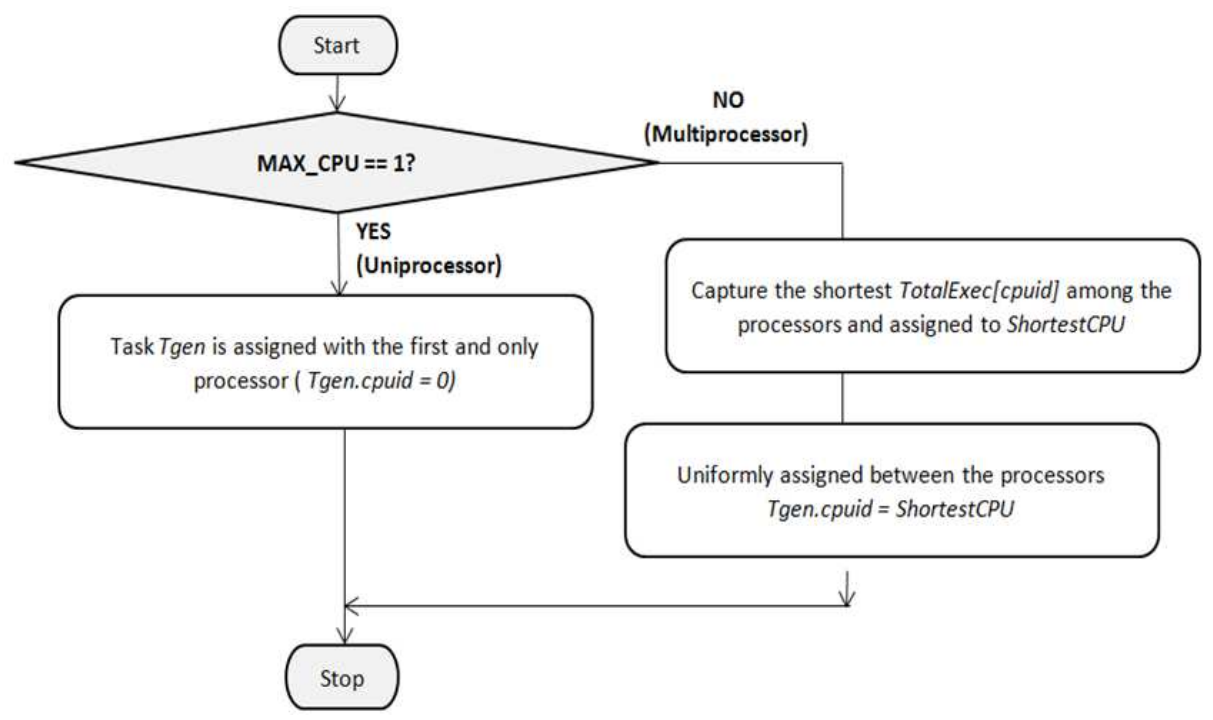

Fig. 3. Task assignment algorithm 


\section{Existing Algorithms}

The features of the existing G-GUA algorithm are simplified as follows:

1. G-GUA uses the PUD metric i.e., task whose execution yields the maximum PUD over others is scheduled in the system. Once a scheduling event is triggered, a new schedule is created that may result in the task executing on the other processors to be preempted.

2. G-GUA allows the request of a task to migrate to the idle resource in another processor in the system. It also allows the request of a task to be inserted into a processor's queue with the least of total remaining execution cost for the respective resource.

The existing NG-GUA algorithm is a TUF/UA multiprocessor real time scheduling algorithm that allows tasks to be subject to run-time uncertainties, overloads and global migration (Garyali et al., 2010). The none greediness in the name of this algorithm describes the tendency of the algorithm to accrue as much total utility during overload situation while in under load situation it uses deadline as its metric and schedule task with the earliest deadline first. The features of the existing NG-GUA are simplified as follows:

1. NG-GUA uses two metrics for task scheduling depending on the overload condition in the system. During overloaded, the PUD metric i.e., task whose execution yields the maximum PUD over others is scheduled in the system. In under load situation, the deadline metric i.e., task with the earliest deadline is scheduled in the system. Once a scheduling event is triggered, a new schedule is created that may result in the task executing on the other processors to be preempted.

2. NG-GUA allows the request of a task to migrate to the idle resource in another processor in the system. It also allows the request of a task to be inserted into a processor's queue with the least of total remaining execution cost for the respective resource.

\section{Proposed Algorithm}

The different approach of GPUAS is in the decision for queuing the requesting task with the lowest PUD in the system. GPUAS uses the lowest PUD metric as opposed to G-GUA that used the least sum of total remaining execution cost for the respective resource. GPUAS uses only the PUD metric to schedule the incoming task as opposed to the existing algorithms that uses dual metrics i.e., PUD and deadline to schedule tasks in the system according to the current load in the system. Figure 4 shows the different between these algorithms to identify the most suitable queue to insert the requesting task i.e., Treq when it cannot be instantaneously scheduled to use a resource in the system. There are two parameters being used by the scheduling algorithms on the decision to locate task Treq as follows:

i. NG-GUA, G-GUA and GPUAS measure the least sum of remaining execution cost for resource rid among the utlist queues. The LeastTime parameter is used to represent the least sum of remaining execution cost. Additionally, the LeastUtlist parameter is used to represent which utlist queue possess the least sum of remaining execution cost. The remaining execution cost for a resource i.e., rid in a queue is captured from the remaining HoldTime parameter of each task that requesting for resource $r i d$ in the respective $u t l i s t$ queue. The sum of the remaining HoldTime of each identified request is accumulated in the TotalCost parameter. Therefore, before the searching procedure for a request in any utlist queue is executed, the value of TotalCost is initialized to 0.0000 as depicted in Fig. 4.

ii. GPUAS additionally measures the highest PUD of task request for the resource rid in the respective utlist queue. The HighestPUD parameter is used to represent the highest PUD among the task request in the respective queue. Initially, this parameter is set to 0.0000 as shown in Fig. 4.

Referring to Fig. 4, the TotalCost and HighestPUD parameter is initialized to 0.0000 . The identification of which utlist queue for the respective rid is depicted in the in res[rid].cpuid parameter. The scheduler search for a request for resource rid in a queue. Two additional pointers are needed for the searching procedure i.e., the work and prev_utlist pointers. The work pointer moves from one element to the next element starting from the head_utlist to the tail_utlist searching for the corresponding request. The prev utlist points to the previous element before the currently processing element that is shown by the work pointer. Initially both of these pointers point to the first element in the utlist queue as depicted in Fig. 4. The work pointer then checks the resource rid of the first element in the utlist queue. If it does not discover the request for resource rid, it will search for the next element. This is repeated to the subsequent elements until the end of the utlist queue.

As shown in Fig. 4, in the case the work pointer discovers a request for rid, the information of the task request is obtained from the tid element of the work pointer i.e., work-tid. For the purpose of clarification, the respective task is known as Twait in the figure. The execution mode of task Twait in Twait.Mode is checked. Subsequently, the execution mode for task Twait is classified into the NORMAL or ABORT mode as stated below: 
i. Task Twait is currently executing in the NORMAL mode. In this case, the scheduler measures the least sum of remaining execution cost for resource rid in the respective utlist queue. The remaining execution cost for resource rid in a utlist queue is captured from the remaining HoldTime parameter obtained from the work->HoldTime parameter. The sum of remaining HoldTime of each identified request is accumulated in the TotalCost parameter. Referring to Fig. 4, the PUD of this task request is calculated as Twait.PUD. The calculation formula of PUD is elaborated in (Jensen et al., 1985; Li et al., 2006). Subsequently, the PUD is then compared with the HighestPUD parameter that contains the value that is currently producing highest PUD among the tasks in the respective utlist queue. Initially, the value of largest PUD is set to 0.0000 . If task Twait produces a larger PUD than the value currently in HighestPUD, the Twait.PUD is considered as the highest PUD so far. Thus, the value of Highest $P U D$ is updated to be equal to the Twait.PUD.

ii. Task Twait is currently executing in the ABORT mode. In this case, the request of task Twait for resource rid in the respective utlist queue can be delayed. Thus, the AbortTime is not accumulated in the TotalCost parameter. The PUD of Twait is equal to 0.0000 . The scheduler then proceed searching for the next element in the respective utlist. The above mentioned procedure is repeated to the subsequent elements until to the end of the utlist. The outcome of this procedure is to obtain the value of TotalCost and HighestPUD parameters.

The measurement in GPUAS and the existing NGGUA and G-GUA algorithms differ as follows:

i. NG-GUA and G-GUA algorithms use the measured TotalCost parameter and compares to the LeastTime parameter. Note that the LeastTime parameter is used to represent the least sum of remaining execution cost among the utlist queues for resource $R$. If the value of LeastTime exceeds the TotalCost parameter, the LeastTime is updated to reflect the least sum of total remaining execution cost among the utlist queues in the system. The LeastUtlist parameter specifies which utlist that contained the least sum of total remaining execution cost i.e., res[rid].cpuid.

ii. GPUAS uses two parameters i.e., the TotalCost and HighestPUD to decide which utlist queue to locate task Treq.

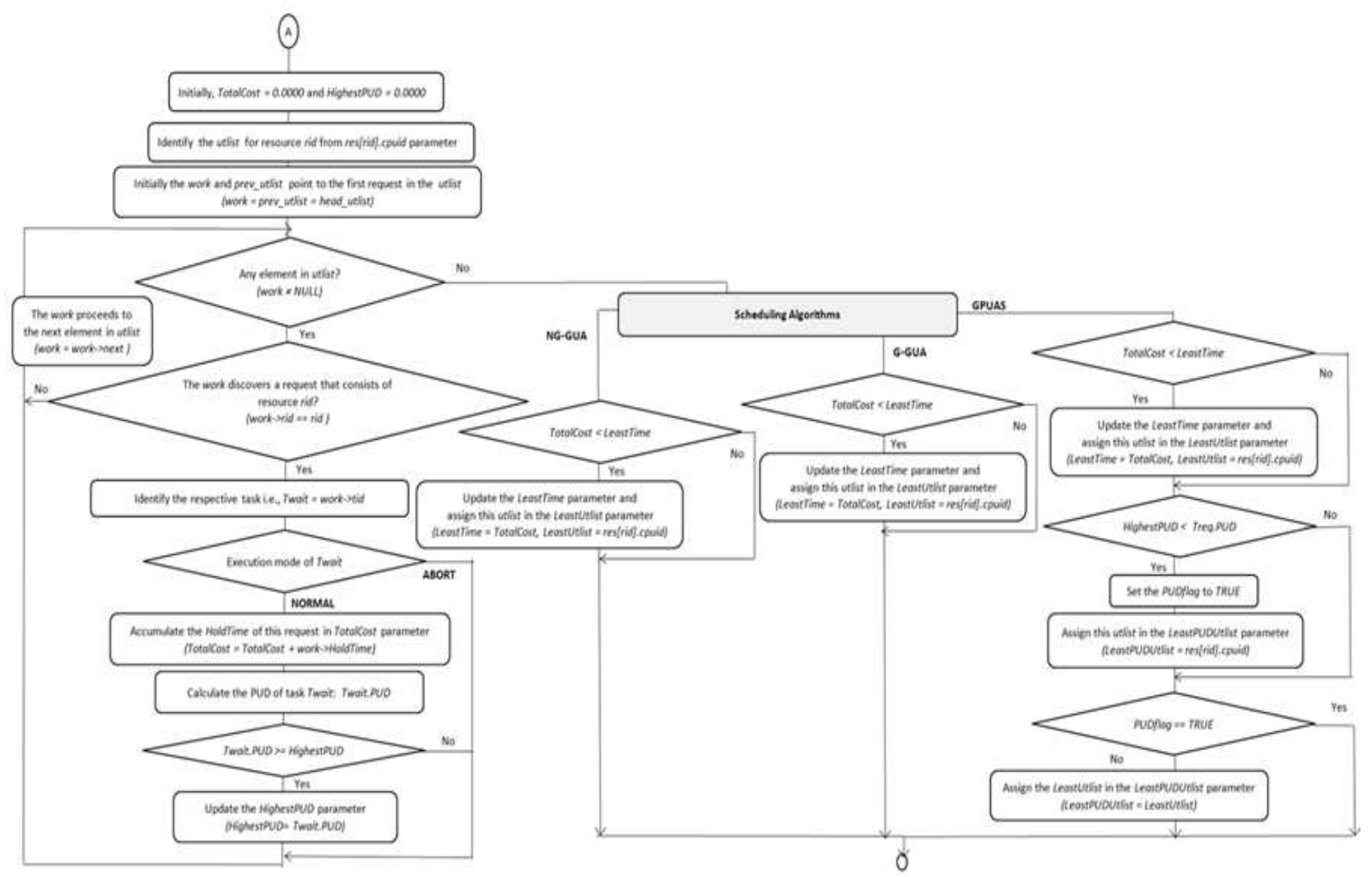

Fig. 4. Scheduling algorithms 
There are three (3) conditions to validate each $u t l i s t$ as stated below:

a. GPUAS firstly identifies the LeastUtlist queue that is identical to the above mentioned G-GUA. The calculated TotalCost parameter is compared to the LeastTime parameter. If the value of LeastTime exceeds the TotalCost parameter, the LeastTime is updated to reflect the least sum of total remaining execution cost among the utlist queues in the system. The LeastUtlist parameter specifies which utlist that contained the least sum of total remaining execution cost i.e., res [rid].cpuid.

b. GPUAS compares the highest PUD in this utlist (i.e., HighestPUD) with the PUD of the requesting task Treq (i.e., Treq.PUD). If the value in Treq.PUD exceeds the HighestPUD parameter, the PUDflag parameter is tagged as TRUE to reflect the existence of a utlist with a lower PUD compared to task Treq. The LeastPUDUtlist parameter specifies which utlist that contained a lower PUD compared to Treq i.e., res[rid].cpuid. Referring to Fig. 4, in the case the value in Treq.PUD is less than or equal to the HighestPUD parameter, the PUDflag value remained unchanged.

c. GPUAS considers the LeastUtlist parameter in the case none of the task in utlist queue produced a lower PUD as compared to the PUD of task Treq. In this case, GPUAS considers the LeastUtlist as its LeastPUDUtlist.

The rationale to select the LeastPUDUtlist queue that has a lower PUD as compared to the requesting task i.e., Treq in GPUAS is to ensure that if task Treq is to be inserted into the utlist queue, the condition in the LeastPUDUtlist will ensure that task Treq can use the resource rid soon after the owner task has releases it.

\section{Experimental Setting}

The performances of real time scheduling algorithms are measured by the metrics which rely on the respective application specifications. The Accrued Utility Ratio (AUR) metric defined in (Jensen et al., 1985) has been extensively utilized in the existing TUF/UA scheduling algorithms and is considered as the standard metric in this domain (Wu et al., 2004; Li et al., 2006).

AUR is defined as the ratio of accrued aggregate utility to the maximum possibly attained utility. Equation 1 shows that each task $i$ has its maximum value of utility which is denoted as MaxAU(i). After a task $\mathrm{i}$ has completed its execution, it will yield a value denoted as Util(i). These values are then accumulated for all tasks i.e., MAX TASKS. The AUR is calculated as:

$$
A U R=\frac{\sum_{i=1}^{\text {MAX_tASKS }} \operatorname{Utill}(i)}{\sum_{i=1}^{M A X_{-} \text {TASKS }} \operatorname{MaxAU}(i)}
$$

\section{Results}

Based upon the results acquired from the simulation, the interpretations of the results are performed. The numbers of processors considered in the system are two, four and eight (Garyali et al., 2010). The scheduling algorithm that is proposed in the multiprocessor scheduling environment is known GPUAS. The NGGUA and G-GUA algorithms are used to compare the performance of GPUAS algorithm. The plots from all the results cover an average load in the range of [1-10] in the multiprocessor environment with two, four and eightcore platform (Garyali et al., 2010) for step and arbitrary TUF task model.

Figure 5 depicts the AUR result under an increasing load for step TUF. From the overall results, as the number of processors increase, a higher utility is recorded for all scheduling algorithms. Overall, the nature of the curves indicates that the proposed GPUAS algorithm has achieved better performance by producing a higher accrued utility as compared to the existing NGGUA and G-GUA algorithms.

Figure 6 depicts the AUR results for execution of the arbitrary TUF tasks in the system. Overall, the patterns of the curves from the results in the arbitrary TUF tasks set are similar to the step TUF tasks set. In the case of arbitrary TUF, a task may not be able to accrue its maximal possible utility even though the execution is completed before its termination time. Although these algorithms guarantee that the highest PUD task to be selected, it does not necessarily represent that the maximum possible utility gained by the executed tasks.

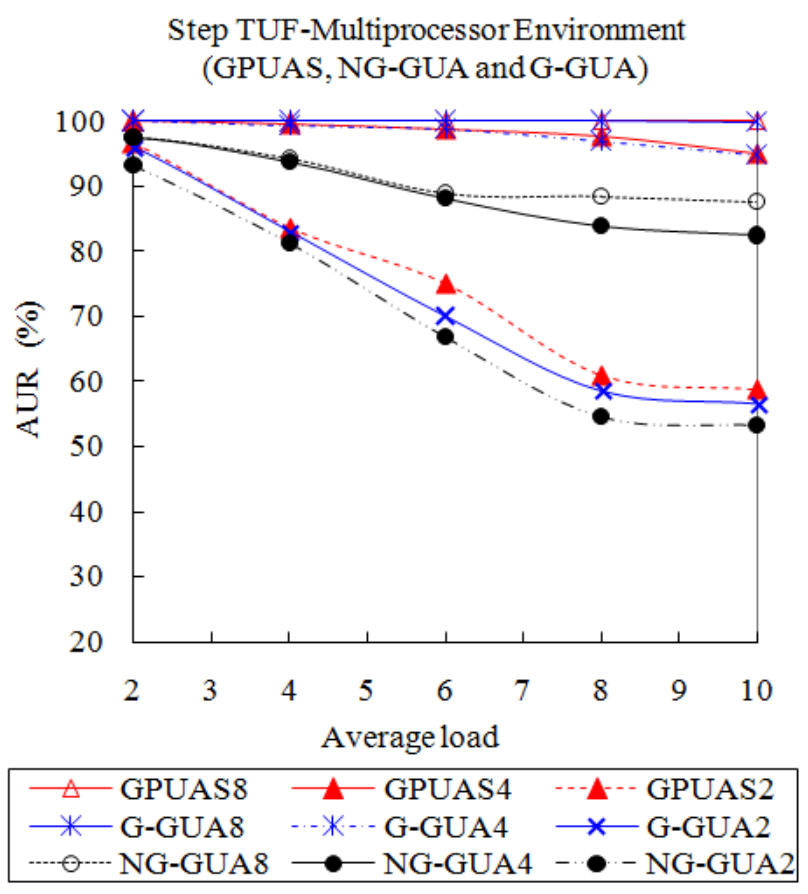

Fig. 5. Results for step TUF 


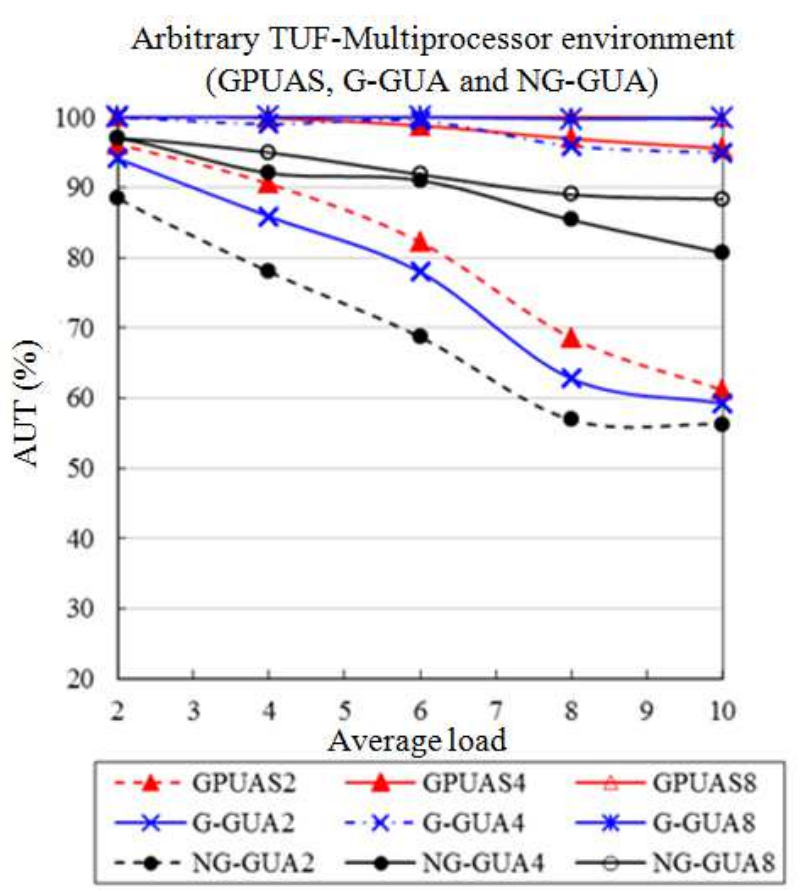

Fig. 6. Results for arbitrary TUF

From the overall results, as the number of processors increase, a higher utility is recorded for all scheduling algorithms. From the overall results, GPUAS shows significant improvement compared to NG-GUA algorithm in all multicore platforms. GPUAS also shows a significant improvement compared to the G-GUA algorithm in the dual core platform. From the results, it is observed that the GPUAS8 and G-GUA8 algorithms that are executed in the eight core platform have produced the highest utility to the system as compared to the dual and quad core platforms.

\section{Discussion}

From the overall results shown in Fig. 5, GPUAS shows significant improvement compared to NG-GUA algorithm in all multicore platforms for the entire load range. However, GPUAS shows a significant improvement compared to the G-GUA algorithm only in the dual core platform. In dual core platform, the average load of 2 is estimated as the starting point of an overloaded situation in the system. At this load, GPUAS2 achieved $96.58 \%$ and G-GUA2 algorithm achieved $96.05 \%$ and NG-GUA2 with $93.14 \%$ of the accumulated utilities. At this load, GPUAS2 has improved the NGGUA2 algorithm for 3.44\% and improved G-GUA2 for $0.53 \%$ of the accumulated utilities.

However, as the load increases, more significance of GPUAS2 is observed in the system as compared to the G-GUA and NG-GUA algorithms. When load is equal to
6 , in the dual core platform, GPUAS2 accrued $75.03 \%$, G-GUA2 accrued $70.05 \%$ and NG-GUA2 accrued $66.76 \%$ of utilities. GPUAS2 algorithm has improved GGUA2 for $4.98 \%$ and outperformed NG-GUA2 algorithm for $8.27 \%$ of the accumulated utilities. At the highest load (i.e., load $=10$ ), GPUAS2 has achieved $58.89 \%$, G-GUA2 with $56.61 \%$ and NG-GUA2 gained $53.24 \%$ of the accumulated utilities. Thus, GPUAS2 has improved G-GUA2 for $2.28 \%$ and outperformed NGGUA2 algorithm for $5.65 \%$. GPUAS2 outperformed the G-GUA2 algorithm because GPUAS2 ensure that the newly request is inserted into the utlist queue that has the highest possibility to be executed as soon as the owner task releases the respective resource. Therefore, the requesting tasks may produce positive utility to the system. On the other hand, G-GUA2 locates the requesting task into the utlist that has the least remaining execution cost. Therefore, G-GUA2 does not guarantee that the requesting task will be scheduled to use the respective resource as soon as the task being scheduled in GPUAS. Therefore, more requesting tasks are ending up waiting in the utlist without being scheduled in GGUA2. This reflects the lower utility accrued in GGUA2 as compared to GPUAS2 in dual core platform. The improvement of GPUAS2 is small i.e., at most only $4.98 \%$ because in the dual core platform, although more number of tasks inserted into the selected queues in GPUAS2 but more tasks are overdue and therefore ending up being aborted. This is why the task placement in GPUAS2 is less over the G-GUA2.

It is observed that GPUAS2 outperformed NG-GUA2 for the entire load range. The excellent performance of GPUAS2 is also observed over the NG-GUA2 for the entire load range. This is because GPUAS2 is a greedy scheduling algorithm and uses PUD as a metric to achieve the highest accrued utility at any instance while NG-GUA2 uses the deadline metric during under load and PUD during overloaded conditions. On the other hand, during overloads NGGUA aborts any requesting task that produced lowest PUD to overcome the overloaded situation. The abortions reduced the value of utility accrued to the system in NG-GUA2. GPUAS omits the abortion and inserts the lowest PUD task into a queue.

From Fig. 5, it is observed that a sharper degradation as the load increases in dual core platform. Although algorithms allows tasks to migrate to the available resources or move to the resources with the least PUD in the utlist queue, in dual core platform the number of available resources is limited. Due to the limited resources, many backlogged tasks are ending up not been migrated anywhere although these algorithms allows them to do so. Due to the limited resources, more tasks are overdue and therefore ending up being aborted. More aborted tasks are produced as the load increases and consequently produced more zero utility tasks to the 
system. This is why a sharper degradation is observed as the load increases in the dual core platform.

Referring to Fig. 5, in four core platform, approximately the system is considered to be overloaded when the average load is equal to 4 . At this load, GPUAS4 has successfully gained $99.63 \%$ of utility and G-GUA4 moderately accrued $99.33 \%$ while NG-GUA4 accrued $93.84 \%$ of the utilities. Thus, the GPUAS4 algorithm outperforms G-GUA4 for $0.30 \%$ and NG-GUA for $5.79 \%$ at this load. It is observed that G-GUA and GPUAS accrued the same utility in the quad and eight core platforms. At the highest load (i.e., load $=10$ ), GPUAS4 has achieved 94.96\%, G-GUA4 with $94.71 \%$ and NG-GUA4 gained $82.42 \%$ of the accumulated utilities. Thus, GPUAS4 has improved GGUA4 for $0.25 \%$ and outperformed NG-GUA4 algorithm for $12.54 \%$.

From the results in Fig. 5, it is observed that the GPUAS8 and G-GUA8 algorithms that are executed in the eight core platform have produced the highest utility to the system as compared to the dual and quad core platforms. In eight core platform, approximately the system is considered to be overloaded when the average load is equal to 8 . At this load, GPUAS8 and G-GUA8 have successfully gained $100 \%$ of utility and NG-GUA moderately accrued $88.45 \%$ of the utilities. Thus, the GPUAS8 and G-GUA8 algorithms outperformed NG-GUA8 for $11.55 \%$ at this load. From the figure, at the highest load (i.e., load = 10), GPUAS 8 and NG-GUA8 have achieved $100 \%$ and NGGUA8 with $87.56 \%$ of the accumulated utilities. Thus, GPUAS8 and G-GUA8 have improved NG-GUA8 for $12.44 \%$. As the number of processors increase, the higher utility accrued to the system by the GPUAS algorithm as compared to the existing G-GUA and NGGUA algorithms. The enhancement of GPUAS has tremendously improved the utility accrued to the system in multiprocessor environment.

Overall, the improvement of GPUAS over the GGUA algorithm is observed only in the dual core platform. In the quad and eight core platforms, the performances of these algorithms are similar. Note that, GPUAS ensures that a task is inserted into a selected queue that has the highest possibility to be executed. In dual core platform, due to limited resources more number of tasks inserted into the selected queues. In dual core platform, only two queues are available for the insertion of tasks in the queues. Therefore, the insertion always occurs between these queues and more number of tasks inserted into the queues. Hence, the PUD metric used in GPUAS for selection of queues for task insertion has an impact to the system. On the other hand, in the quad and eight core platforms, more resources are available. Therefore, less number of tasks inserted into the queues. Therefore, the metric used for task insertion in GPUAS has a minor impact to the system.

\section{Conclusion}

This paper has discussed the design and evaluation of GPUAS in the multiprocessor environment. The proposed GPUAS algorithm is compared with the existing NG-GUA and G-GUA algorithms. Simulation results revealed that GPUAS2 has improved for less than $4.98 \%$ on G-GUA in dual core platform and remain the same performances in quad and eight core platforms. Since GPUAS has improved G-GUA in the metric used for insertion of task in a queue, GPUAS only has impact on the system with less number of queue i.e., in the dual core platform with only two queues available. In the quad and eight core platforms, more resources are available and therefore less number of tasks is inserted into a queue. Therefore insertion procedure is less significant in this environment. Simulation results also revealed that GPUAS has improved the NG-GUA at most $12.44 \%$ for the entire load range in all platforms. This is because GPUAS omits the unnecessary abortions that occur in NGGUA. Overall, the GPUAS algorithm outperforms the existing algorithms by accruing the highest utility to the system due to the highest resource consumption by exploiting the migration and task insertion attributes of the executed tasks. This chapter also has confirmed the advantage of GPUAS as compared to the existing NGGUA algorithm in all platforms for at most $12.44 \%$ and has improved the G-GUA algorithm at most $4.98 \%$ in the dual core platform. The contribution of GPUAS algorithm in the dual, quad and eight core platforms that achieved the highest accrued utility and success ratio making it suitable and efficient scheduling algorithm for real time application.

\section{Acknowledgement}

The authors wish to thank anonymous reviewers for their valuable, detailed comments that improve both the content and representation of this study.

\section{Funding Information}

This research was funded by the Ministry of Higher Education Malaysia and Universiti Putra Malaysia under Fundamental Research Grant FRGS 08-01-15-1722FR

\section{Author's Contributions}

All authors equally contributed in this work.

\section{Ethics}

The corresponding author confirms that the other authors have read and approved the manuscript and there is no ethical issue involved. This paper is original and contains unpublished material. 


\section{References}

Garyali, P., M. Dellinger and B. Ravindran, 2010. On best-effort utility accrual real-time scheduling on multiprocessors. Proceedings of the 14th International Conference on Principles of Distributed Systems, Dec. 14-17, Springer-Verlag Berlin, Heidelberg, pp: 270-285.

DOI: $10.1007 / 978-3-642-17653-121$

Idawaty, A., S. Shamala, M. Othman and Z. Zuriati, 2012. Performance of partition utility accrual real time scheduling algorithm. J. Comput. Sci., 8: 1225-1234.

Jensen, E.D., C.D. Locke and H. Tokuda, 1985. A timedriven scheduling model for real-time operating systems. Proceeding of the IEEE Symposium on Real-Time System, (SRS' 85), IEEE Computer Society, pp: 112-122.
Li, P., H. Wu, B. Ravindran and E.D. Jensen, 2006. A utility accrual scheduling algorithm for real-time activities with mutual exclusion resource constraints. IEEE Trans. Computer., 55: 454-469. DOI: $10.1109 /$ TC.2006.47

Wu, H., B. Ravindran, E.D. Jensen and P. Li, 2004. CPU scheduling for statistically-assured real-time performance and improved energy efficiency. Proceeding of the 2nd IEEE/ACM/IFIP International Conference on Hardware/Software Codesign and System Synthesis, Sept. 8-10, IEEE Xplore Press, USA, pp: 110-115.

DOI: $10.1109 /$ CODESS.2004.240827 